\title{
Changes in MMP-2, MMP-9, inflammation, blood coagulation and intestinal mucosal permeability in patients with active ulcerative colitis
}

\author{
XUESONG BAI, GUANG BAI, LIDONG TANG, LIN LIU, YUFENG LI and WEI JIANG \\ Department of Gastroenterology, Hospital of Liaoning University of \\ Traditional Chinese Medicine, Shenyang, Liaoning 110032, P.R. China \\ Received October 3, 2019; Accepted February 4, 2020
}

DOI: $10.3892 /$ etm. 2020.8710

\begin{abstract}
Changes in matrix metalloproteinase (MMP)-2, MMP-9, inflammation, blood coagulation factors and intestinal mucosal permeability in patients with active ulcerative colitis (UC) were investigated. A total of 50 active UC patients treated in our hospital from January 2016 to December 2018 were selected as the UC group, whereas 50 normal subjects receiving physical examination were selected as the control group. Venous blood was drawn to detect the content of early predictors, C-reactive protein (CRP), follistatin-like protein 1 (FSTL1) and D-dimer in serum. The disease activity index (DAI) score was recorded in both groups, the levels of MMP-2 and MMP-9 were determined, and the inflammatory factors interleukin (IL)-1, IL-6 and tumor necrosis factor (TNF)- $\alpha$ were also detected. Moreover, the blood coagulation factors, platelet count, prothrombin time (PT), activated partial thromboplastin time (APTT) and fibrinogen level were detected, the content of lactulose (L) and mannitol (M) in the urine after oral administration of $\mathrm{L}$ and $\mathrm{M}$ test liquid was determined via high-performance liquid chromatography in both groups, and the L/M ratio was calculated. In UC group, the content of CRP, FSTL1 and D-dimer was significantly higher than that in the control group $(\mathrm{P}<0.05)$. The DAI score was significantly higher $(\mathrm{P}<0.05)$, the content of MMP-2 and MMP-9 was remarkably raised $(\mathrm{P}<0.05)$, the platelet count, PT, APTT and fibrinogen level were all obviously increased $(\mathrm{P}<0.05)$, and the $\mathrm{L} / \mathrm{M}$ ratio was notably lower $(\mathrm{P}<0.05)$ in the UC group than in the control group. In patients with active UC, MMP-2, MMP-9 and inflammatory factors were significantly increased, and there were changes in the blood coagulation
\end{abstract}

Correspondence to: Dr Xuesong Bai, Department of Gastroenterology, Hospital of Liaoning University of Traditional Chinese Medicine, 33 Beiling Street, Huanggu, Shenyang, Liaoning 110032, P.R. China

E-mail:1mxsu091@163.com

Key words: active phase, ulcerative colitis patients, MMP-2, MMP-9, inflammatory factors, blood coagulation factors, intestinal mucosal permeability factors and intestinal mucosal permeability, which further promote the occurrence and development of UC.

\section{Introduction}

Ulcerative colitis (UC), also known as non-specific UC, mainly characterized by erosion and ulcers, is a periodic lifelong disease. The main manifestations of UC are severe abdominal pain, weight loss, intestinal inflammation, rectal bleeding, diarrhea, dehydration and tenesmus. The etiology and pathogenesis of this disease are complicated and have not been fully elucidated yet. The morbidity rate of UC has an obvious increasing trend in China, and the patients have been gradually younger. The occurrence and development of UC have serious affects on the human health and quality of life, and therefore UC is listed by the World Health Organization as one of the refractory diseases of the modern world (1-3). In UC and other inflammatory bowel diseases, damage of intestinal mucosal barrier, immune cell dysfunction and intestinal neuronal dysfunction occur. Currently, the main drugs used for UC include glucocorticoids, immunosuppressants and tumor necrosis factor (TNF) (4), which have certain effects, but also great side-effects, and the recurrence rate and treatment expenses are high. Most scholars argue that persistent intestinal infection, intestinal mucosal barrier defect, intestinal mucosal immunoregulatory abnormality, genetic and environmental factors are jointly involved in the occurrence of UC $(5,6)$. UC is a frequently occurring inflammatory bowel disease and its morbidity rate has increased significantly in recent years worldwide. Studies have shown that the morbidity rate of UC remains at a high level in developed countries, whereas it obviously increases in developing countries or regions previously having a lower morbidity rate, making UC a global disease $(7,8)$. The complicated pathogenesis of UC cannot be clarified by research based on 'immune-mediated inflammatory response' ideas, leading to limited diagnosis and treatment means. Therefore, studying and developing new treatment means is imperative.

Matrix metalloproteinase (MMP)-2 and MMP-9 are two types of MMPs (9-11). According to previous studies, MMP-2 and MMP-9 are actively involved in the pathophysiological processes in patients with inflammatory bowel diseases (12-14). Studies have shown that MMP-2 and MMP-9 affect the tight 
junctions among mucosal cells, increase the intestinal mucosal permeability and aggravate the impairment of mucosal barrier function. After three cycles of drug treatment of UC, the levels of MMP-2 and MMP-9 decline repairing the intestinal mucosal damage and reducing the incidence of inappetence, nausea, vomiting and mucositis $(15,16)$. Therefore, effectively controlling the content of MMPs is important for the treatment of UC.

Studies have shown that UC patients have hypercoagulability, hyperfunction of the fibrinolytic system, increased prothrombin time (PT) and activated partial thromboplastin time (APTT), and changes in intestinal mucosal permeability, which can be used as indexes for evaluating the UC activity $(17,18)$. In the present study, UC patients and normal subjects receiving physical examination were enrolled. The content of early predictors, C-reactive protein (CRP), follistatin-like protein 1 (FSTL1) and D-dimer in the serum was detected, the disease activity index (DAI) score was recorded, the content of MMP-2 and MMP-9 was determined, and the inflammatory factors interleukin (IL)-1, IL-6 and TNF- $\alpha$ were also detected. Moreover, the blood coagulation factors, platelet count, PT, APTT and fibrinogen level were detected, and the mucosal permeability in terms of the lactulose (L)/mannitol (M) ratio was calculated, aiming to explore the pathogenesis of UC and provide theoretical and experimental bases for the treatment and prognosis of UC patients.

\section{Subjects and methods}

General data. A total of 50 active UC patients treated in the Hospital of Liaoning University of Traditional Chinese Medicine (Shenyang, China) from January 2016 to December 2018 were selected as the UC group, whereas another 50 healthy subjects receiving physical examination were selected as the control group. Inclusion criteria: Patients diagnosed with UC via intestinal microscopy, those who voluntarily participated in the study and signed the informed consent, those who had received no treatment before, and those with DAI score $>2$ points. Exclusion criteria: Patients with bacterial colitis caused by Salmonella, hemorrhagic necrotic enteritis, secondary infection complicated with severe renal or hepatic dysfunction, colorectal cancer or liver cancer. All clinical specimens in this experiment were collected upon the agreement of the Ethics Committee of the Hospital of Liaoning University of Traditional Chinese Medicine and the family members. The study was approved by the Ethics Committee of the hospital and signed written informed consents were obtained from all participants or their guardians before the study. The specific clinical data, including age, sex, body weight and disease severity of patients were collected at the time of admission (Table I).

DAI score. The DAI scoring includes the evaluation of hemafecia, diarrhea, mucosal manifestations and disease conditions. Hemafecia: None (0 point), a little (1 point), obvious ( 2 points), and frequent ( 3 points). Diarrhea: Normal (0 point), once to twice a day (1 point), 3-4 times a day (2 points), and 5 times or more ( 3 points). Mucosal manifestations: Normal ( 0 point), mildly brittle (1 point), moderately brittle ( 2 points), and severely brittle with exudation (3 points). Disease conditions: Normal ( 0 point), mild (1 point), moderate ( 2 points), and severe (3 points). The DAI score is the sum of the scores in all
Table I. Clinical characteristics of the study subjects.

\begin{tabular}{lcc}
\hline Characteristics & Control group & UC group \\
\hline Sample size & 50 & 50 \\
Males & 24 & 25 \\
Mean age (years) & $40 \pm 10$ & $39 \pm 9$ \\
Mean weight $(\mathrm{kg})$ & $47 \pm 9$ & $49 \pm 10$ \\
BMI $\left(\mathrm{kg} / \mathrm{m}^{2}\right)$ & $21.5 \pm 1.0$ & $21.2 \pm 0.9$ \\
Severe cases & 0 & 25 \\
Moderate cases & 0 & 25 \\
\hline
\end{tabular}

UC, ulcerative colitis.

four categories and was recorded in detail by special personnel for subsequent study and analysis.

Detection of serum CRP, FSTL1 and D-dimer. In this study, $5 \mathrm{ml}$ of venous blood were drawn from the arm of the study subjects to detect the content of CRP, FSTL1 and D-dimer, in order to predict the development of the disease in advance. The blood was inserted into 5-ml Eppendorf (EP) tubes containing anticoagulant and placed at room temperature for $20 \mathrm{~min}$, followed by centrifugation at $4^{\circ} \mathrm{C}, 2,000 \mathrm{xg}$ for $15 \mathrm{~min}$. Next, the supernatant was collected to detect the changes in the content of CRP, FSTL1 and D-dimer via enzyme-linked immunosorbent assay (ELISA), providing an important theoretical reference for early detection of UC. CRP kit (cat. no. H126), FSTL1 kit (cat. no. E027-1-3) and D-dimer kit (cat. no. E029-1-1) were all purchased from Nanjing Jiancheng Bioengineering Institute.

Detection of serum inflammatory factors via ELISA. A total of $5 \mathrm{ml}$ of venous blood were drawn from the arm of the study subjects, placed into EP tubes containing anticoagulant and centrifuged at 2,000 $\mathrm{x} \mathrm{g}$ at room temperature for $15 \mathrm{~min}$. Next, the supernatant was collected to detect the serum inflammatory factors IL-6, IL-1 and TNF- $\alpha$ following the manufacturer's instructions of the ELISA kits (Nanjing Jiancheng Bioengineering Institute). IL-6 kit (cat. no. H007), IL-1 kit (cat. no. H002) and TNF- $\alpha$ kit (cat. no. H052) were all purchased from Nanjing Jiancheng Bioengineering Institute. Finally, the absorbance in each group was detected using a microplate reader.

Detection of content of plasma MMP-9 and MMP-2. A total of $5 \mathrm{ml}$ of fasting venous blood were drawn early in the morning from the elbow of the study subjects and centrifuged at $2,000 \mathrm{x} \mathrm{g}$ at room temperature for $15 \mathrm{~min}$. Next, the supernatant was collected to detect the levels of plasma MMP-9 and MMP-2 using double-antibody sandwich ELISA according to the manufacturer's instructions. Finally, the absorbance in each group was detected using a microplate reader. MMP-9 kit (cat. no. H146-4) and MMP-2 kit (cat. no. H146-1) were purchased from Nanjing Jiancheng Bioengineering Institute.

Detection of blood coagulation function. Fasting peripheral venous blood was drawn early in the morning from all the study subjects, inserted into anticoagulant tubes with $0.2 \mathrm{ml}$ 


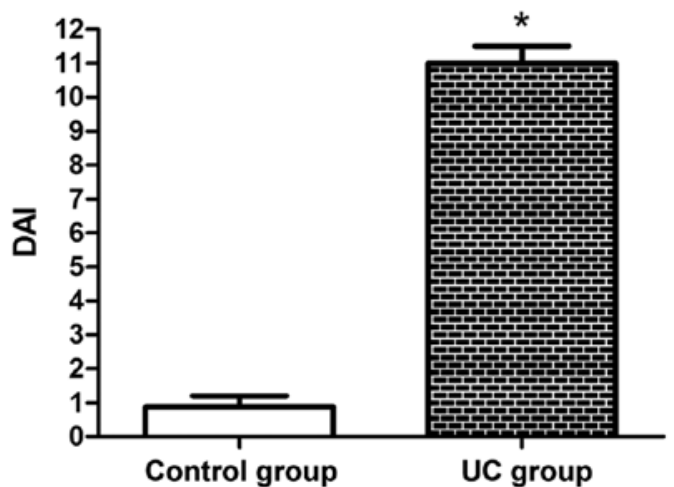

Figure 1. DAI score was basically close to 1 in the control group and was significantly higher in the UC group compared with that in the control group ${ }^{*} \mathrm{P}<0.05$ vs. control group. DAI, disease activity index; UC, ulcerative colitis.

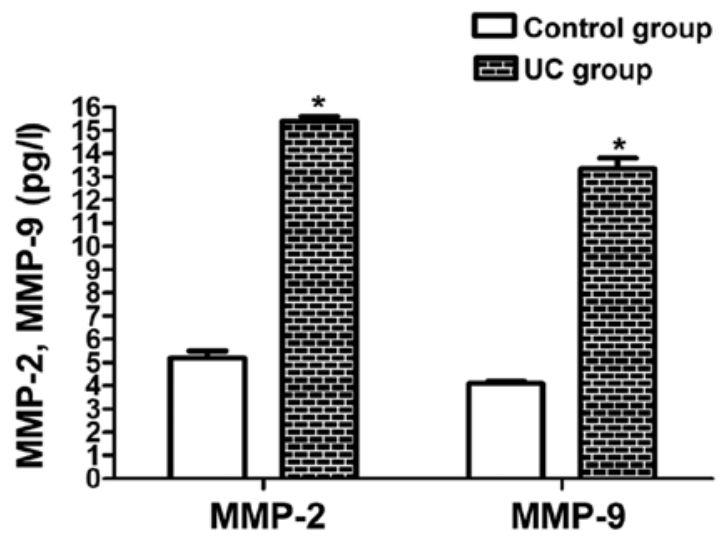

Figure 2. The content of plasma MMP-2 and MMP-9 was markedly raised in the UC group compared with that in the control group. ${ }^{*} \mathrm{P}<0.05$ vs. control group. MMP, matrix metalloproteinase; UC, ulcerative colitis.

of sodium citrate, and placed at room temperature for $20 \mathrm{~min}$, followed by centrifugation at $4^{\circ} \mathrm{C}, 2,000 \mathrm{xg}$ for $10 \mathrm{~min}$. Next, the separated plasma was collected to determine the platelet count, PT, APTT and fibrinogen level within $24 \mathrm{~h}$ using a full-automatic biochemical analyzer (BS-220; Shenzhen Mindray Bio-Medical Electronics Co., Ltd.).

Determination of intestinal mucosal permeability. The concentration of $\mathrm{L}$ and $\mathrm{M}$ in the urine was measured via Waters 515 high-performance liquid chromatography (Waters Corporation). Alltima-NH2 Column (Alltech Medical Systems, LLC) was used. The mobile phase was acetonitrile-water (67:33). The flow rate was $1.0 \mathrm{ml} / \mathrm{min}$ and the column temperature was $45^{\circ} \mathrm{C}$. Urine $(1 \mathrm{ml})$ was collected from all subjects into EP tubes, and centrifuged at $4^{\circ} \mathrm{C}, 2,000 \mathrm{xg}$ for $15 \mathrm{~min}$. The supernatant was aspirated, and added with $100 \mathrm{ml}$ of acetonitrile to precipitate the protein. After vortex mixing, the mixture was centrifuged at $4^{\circ} \mathrm{C}, 2,000 \mathrm{x}$ g for $15 \mathrm{~min}$, and the supernatant was aspirated and deionized, followed by vortex mixing and centrifugation at $4^{\circ} \mathrm{C}, 2,000 \mathrm{x} \mathrm{g}$ for $5 \mathrm{~min}$. Next, the supernatant was taken, and filtered using the water-based filter membrane. Finally, the content of $\mathrm{L}$ and $\mathrm{M}$ was detected and the $\mathrm{L} / \mathrm{M}$ ratio was calculated (column temperature, $25^{\circ} \mathrm{C}$; internal heating in differential detector at $32^{\circ} \mathrm{C}$, according to the manufacturer's instructions).
Table II. Content of serum CRP, FSTL1 and D-dimer.

\begin{tabular}{lccc}
\hline Groups & CRP $(\mathrm{mg} / \mathrm{l})$ & FSTL1 $(\mu \mathrm{g} / \mathrm{l})$ & $\mathrm{D}-\operatorname{dimer}(\mathrm{mg} / \mathrm{l})$ \\
\hline Control group & $2.2 \pm 0.2$ & $8 \pm 0.8$ & $0.4 \pm 0.3$ \\
UC group & $10.1 \pm 0.1^{\mathrm{a}}$ & $29 \pm 0.6^{\mathrm{a}}$ & $2.5 \pm 0.4^{\mathrm{a}}$ \\
\hline
\end{tabular}

The content of CRP, FSTL1 and D-dimer was significantly higher in the UC group than that in the control group. ${ }^{\mathrm{a}} \mathrm{P}<0.05$ vs. control group. CRP, C-reactive protein; FSTL1, follistatin-like protein 1; UC, ulcerative colitis.

Table III. Levels of serum IL-1, IL-6 and TNF- $\alpha$.

\begin{tabular}{lccc}
\hline Groups & IL-1 (mg/l) & TNF- $\alpha(\mathrm{fmol} / \mathrm{ml})$ & IL-6 (mg/l) \\
\hline Control group & $20.5 \pm 1.9$ & $14.5 \pm 1.2$ & $24.4 \pm 1.1$ \\
UC group & $43.2 \pm 2.0^{\mathrm{a}}$ & $34.7 \pm 1.1^{\mathrm{a}}$ & $40.1 \pm 1.0^{\mathrm{a}}$
\end{tabular}

The levels of IL-1, IL- 6 and TNF- $\alpha$ were obviously increased in the UC group compared with those in the control group. ${ }^{\mathrm{a}} \mathrm{P}<0.05 \mathrm{vs}$. control group. IL, interleukin; TNF, tumor necrosis factor; UC, ulcerative colitis.

Statistical analysis. All data obtained from the experiments were statistically analyzed using Statistical Product and Service Solutions (SPSS) 21.0 software (IBM Corp.). The experimental results were expressed as the mean \pm standard deviation. Student's t-test (two-tailed) was used for the comparison of variables between two groups. The $\mathrm{L} / \mathrm{M}$ ratios between two groups were compared using Wilcoxon-Mann-Whitney two-tailed test. The bar graphs were plotted using GraphPad Prism 5.0 (GraphPad Software, Inc.). P $<0.05$ was considered to indicate a statistically significant difference.

\section{Results}

DAI score. As shown in Fig. 1, the DAI score was basically close to 1 in the control group, whereas it was higher in the UC group, and there was a significant difference $(\mathrm{P}<0.05)$.

Serum CRP, FSTL1 and D-dimer. The content of serum CRP, FSTL1 and D-dimer can predict the occurrence of UC in advance. As shown in Table II, the content of CRP, FSTL1 and D-dimer in the UC group was markedly higher than that in the control group $(\mathrm{P}<0.05)$, indicating that there are significant changes in the content of the three indexes when UC occurs.

Serum inflammatory factors detected via ELISA. The levels of IL-1, IL-6 and TNF- $\alpha$ were obviously increased in the UC group $(\mathrm{P}<0.05)$, whereas they were normal in the control group (Table III).

Content of plasma MMP-9 and MMP-2. The content of MMP-2 and MMP-9 in the UC group was raised markedly compared with that in the control group $(\mathrm{P}<0.05)$ (Fig. 2), which further promotes the development of UC. 
Table IV. Blood coagulation function.

\begin{tabular}{lcllc}
\hline & $\begin{array}{c}\text { Platelet count } \\
\left(10^{9} / 1\right)\end{array}$ & PT (S) & APTT (S) & $\begin{array}{c}\text { Fibrinogen } \\
\text { level }(\mathrm{g} / \mathrm{l})\end{array}$ \\
\hline Control group & $220 \pm 5$ & $11 \pm 0.8$ & $20 \pm 1.2$ & $3 \pm 0.5$ \\
UC group & $350 \pm 6^{\mathrm{a}}$ & $14 \pm 0.6^{\mathrm{a}}$ & $31 \pm 0.8^{\mathrm{a}}$ & $5 \pm 0.7^{\mathrm{a}}$ \\
\hline
\end{tabular}

Platelet count,PT,APTT and fibrinogen level were evidently increased in the UC group than those in the control group. ${ }^{\mathrm{a}} \mathrm{P}<0.05 \mathrm{vs}$. control group. PT, prothrombin; APTT, activated partial thromboplastin time; UC, ulcerative colitis.

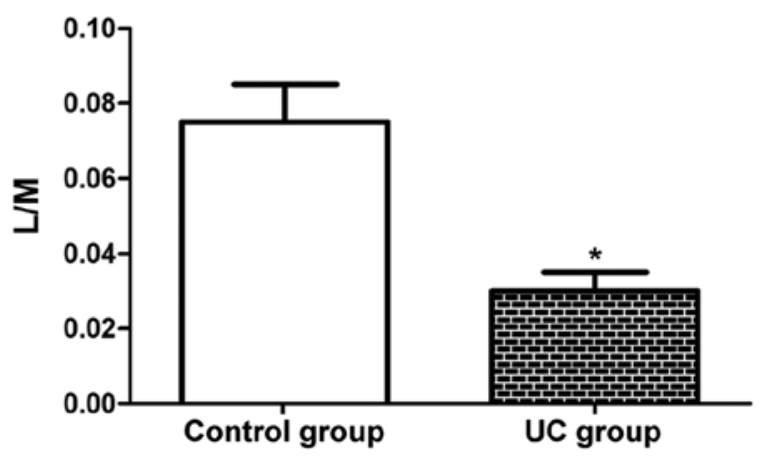

Figure 3. Intestinal mucosal permeability. The L/M ratio was notably lower in the UC group than that in the control group. ${ }^{*} \mathrm{P}<0.05$ vs. control group. L, lactulose; M, mannitol; UC, ulcerative colitis.

Blood coagulation function. The subjects in the UC group had evidently increased platelet count, PT, APTT and fibrinogen level compared with those in the control group $(\mathrm{P}<0.05)($ Table IV), which indicates that the blood coagulation function is changed during UC, further promoting the development of UC.

Intestinal mucosal permeability. As shown in Fig. 3, the $\mathrm{L} / \mathrm{M}$ ratio in the UC group (0.03) was notably lower than that in the control group $(0.07)(\mathrm{P}<0.05)$, which suggests that the intestinal mucosal permeability evidently rises in UC patients, further facilitating the development of the disease.

\section{Discussion}

UC and Crohn's disease are two major inflammatory bowel diseases that share some common features. They can be distinguished by differences in genetic susceptibility, risk factors, and clinical, endoscopic and histological features. The exact pathogenesis of inflammatory bowel diseases remains unclear. Genetically susceptible individuals seemingly have the disordered mucosal immune response, leading to intestinal inflammation. The inflammation of UC is mainly confined to the mucosal surface. UC begins in the rectum and usually extends proximally to the entire colon. However, part of patients with left-sided colitis or proctitis may suffer from cecal dilation $(19,20)$. Based on the degree of colon involvement, proctitis, left-sided colitis and extensive colitis (pancolitis) have different conditions, and they are also difficult to be cured. In the present study, UC patients and normal subjects receiving physical examination were enrolled, and the serum early predictors, including MMP-2, MMP-9, IL-6, TNF- $\alpha$, blood coagulation factors and mucosal permeability indexes were detected, aiming to explore the pathogenesis of $\mathrm{UC}$ and provide theoretical and experimental bases for the treatment and prognosis of UC patients. The content of serum CRP, FSTL1 and D-dimer can predict the occurrence of UC in advance. The detection results revealed that the content of CRP, FSTL1 and D-dimer in the UC group was apparently higher than that in the control group, indicating that there are remarkable changes in these three indexes when UC occurs, which provides a diagnostic basis for the early-onset UC, similar to previous studies $(6,18)$. In addition, DAI was scored in each group. It was found that the DAI score was basically close to 1 in control group, whereas it was generally higher in the UC group, demonstrating that UC patients have severer hemafecia, diarrhea and mucosal manifestations.

Inflammation plays an important role in UC, and inflammatory cytokines have attracted widespread attention. TNF- $\alpha$, one of the major cytokines that mediate the early response to intestinal injury, can stimulate the production of IL-6. Under normal conditions, the concentration of IL-6 in healthy subjects is low or even undetectable. The changes in the concentration of inflammatory factors are related to the duration and severity of UC, and the increases in their levels have been proven to be associated with the raised morbidity rate of UC (21). In the present study, the levels of IL-1, IL-6 and TNF- $\alpha$ were obviously elevated in the UC group, whereas they were normal in the control group. MMPs play important roles in the degradation of ECM and destruction of proteolytic enzymes. Proteolytic enzymes are stimulated by pro-inflammatory cytokines, and fully activated MMPs may aggravate the intestinal inflammatory injury. In addition, some components, such as IL-1, TNF and lipopolysaccharide, can specifically induce the upregulation of MMP-3 and MMP-9, which are important factors for intestinal injury (22). In this study, it was observed that the content of MMP-2 and MMP-9 in the UC group was obviously increased, which further promotes the development of UC. After intestinal injury in UC, the blood coagulation disorder is early detected. The ability to form fibrin clots at the injury site is indispensable for limiting bleeding and subsequent survival. Therefore, the standard coagulation assay, including the detection of PT and APTT, can accurately reflect the blood coagulation function of UC patients (23). In the present study, the patients in the UC group had evidently increased platelet count, PT, APTT and fibrinogen level, which indicates that the blood coagulation function is altered during UC, further promoting the development of UC. The increased L/M ratio corresponds to the enhancement of intestinal mucosal permeability, and the reason is that the damage of intestinal mucosal cells causes atrophy of intestinal mucosa, thus resulting in the increase in intercellular space. Research has shown that glutamine is one of the essential amino acids to maintain the intestinal mucosal barrier, and glutamine nutrition support can reduce intestinal injury. Therefore, inhibiting intestinal mucosal intercellular space, regulating the expression of tight junction protein, and protecting the intestinal mucosal barrier function can gradually lower the L/M ratio (24). In this study, the $\mathrm{L} / \mathrm{M}$ ratio in the UC group was remarkably higher than that 
in the control group, which suggests that the intestinal mucosal permeability evidently rises in UC patients, further facilitating the development of the disease. The aforementioned findings are similar to the research results of Gao et al (25) and Li et al (26). Differently, it was found that the content of MMP-2 and MMP-9 was increased in patients with active UC rather than in patients with Crohn's disease or in animal models. The patients included in our study were all with active UC, whereas the other studies did not emphasize on this point. In addition, up to our knowledge, it is the first time that serum early predictors, DAI scores, MMP-2, MMP-9, inflammatory factors, blood coagulation indexes and the permeability intestinal mucosa are all included in one study, suggesting that UC is a complex disease involving multiple mechanisms. The present study provides a more comprehensive theoretical basis for the pathogenesis, prevention and treatment of UC, as well as new ideas for subsequent further research.

In conclusion, in the present study it was confirmed through a series of experiments that there are changes in the MMPs, inflammatory factors, blood coagulation function and intestinal mucosal permeability in active UC patients, further promoting the development of disease. In the future, such changes can be further verified by animal experiments.

\section{Acknowledgements}

Not applicable.

\section{Funding}

No funding was received.

\section{Availability of data and materials}

All data generated or analyzed during this study are included in this published article.

\section{Authors' contributions}

$\mathrm{XB}, \mathrm{YL}$ and $\mathrm{WJ}$ designed the study and performed the experiments. XB, LT, LL and GB were involved in the conception and design of the study. LT, YL and WJ analyzed the data. $\mathrm{XB}, \mathrm{YL}$ and WJ prepared the manuscript. All authors read and approved the final version of the manuscript.

\section{Ethics approval and consent to participate}

The study was approved by the Ethics Committee of the Hospital of Liaoning University of Traditional Chinese Medicine (Shenyang, China). Signed written informed consents were obtained from all participants or their guardians before the study.

\section{Patient consent for publication}

Not applicable.

\section{Competing interests}

The authors declare that they have no competing interests.

\section{References}

1. Mason A, Malik S, McMillan M, McNeilly JD, Bishop J, McGrogan P, Russell RK and Ahmed SF: A prospective longitudinal study of growth and pubertal progress in adolescents with inflammatory bowel disease. Horm Res Paediatr 83: 45-54, 2015.

2. Sun PL and Zhang S: Correlations of 25-hydroxyvitamin D3 level in patients with ulcerative colitis with inflammation level, immunity and disease activity. Eur Rev Med Pharmacol Sci 22: 5635-5639, 2018.

3. Lamas B, Richard ML, Leducq V, Pham HP, Michel ML, Da Costa G, Bridonneau C, Jegou S, Hoffmann TW, Natividad JM, et al: CARD9 impacts colitis by altering gut microbiota metabolism of tryptophan into aryl hydrocarbon receptor ligands. Nat Med 22: 598-605, 2016.

4. Jørgensen KK, Olsen IC, Goll GL, Lorentzen M, Bolstad N, Haavardsholm EA, Lundin KEA, Mørk C, Jahnsen J, Kvien TK, et al; NOR-SWITCH study group: Switching from originator infliximab to biosimilar CT-P13 compared with maintained treatment with originator infliximab (NOR-SWITCH): A 52-week, randomised, double-blind, non-inferiority trial. Lancet 389: 2304-2316, 2017.

5. Baumgart DC and Sandborn WJ: Crohn's disease. Lancet 380: 1590-1605, 2012.

6. Ordás I,Eckmann L, Talamini M, Baumgart DC and Sandborn WJ: Ulcerative colitis. Lancet 380: 1606-1619, 2012.

7. Ananthakrishnan AN: Epidemiology and risk factors for IBD Nat Rev Gastroenterol Hepatol 12: 205-217, 2015.

8. Mbachi C, Attar B, Oyenubi O, Yuchen W, Efesomwan A, Paintsil I, Madhu M, Ajiboye O, Simons-Linares CR, Trick WE and Kotwal V: Association between cannabis use and complications related to ulcerative colitis inhospitalized patients: A propensity matched retrospective cohortstudy. Medicine (Baltimore) 98: e16551, 2019.

9. Jakubowska K, Pryczynicz A, Iwanowicz P, Niewiński A, Maciorkowska E, Hapanowicz J, Jagodzińska D, Kemona A and Guzińska-Ustymowicz K: Expressions of matrix metalloproteinases (MMP-2, MMP-7, and MMP-9) and their inhibitors (TIMP-1, TIMP-2) in inflammatory bowel diseases. Gastroenterol Res Pract 2016: 2456179, 2016.

10. Khan S, Shukla S, Sinha S, Lakra AD, Bora HK and Meeran SM: Centchroman suppresses breast cancer metastasis by reversing epithelial-mesenchymal transition via downregulation of HER2/ERK1/2/MMP-9 signaling. Int J Biochem Cell Biol 58: $1-16,2015$.

11. Giannopoulos G, Pavlakis K, Parasi A, Kavatzas N, Tiniakos D, Karakosta A, Tzanakis N and Peros G: The expression of matrix metalloproteinases-2 and -9 and their tissue inhibitor 2 in pancreatic ductal and ampullary carcinoma and their relation to angiogenesis and clinicopathological parameters. Anticancer Res 28B: B1875-B1881, 2008.

12. Jakubowska K, Pryczynicz A, Januszewska J, Sidorkiewicz I, Kemona A, Niewiński A, Lewczuk Ł, Kęra B and GuzińskaUstymowicz K: Expressions of matrix metalloproteinases 2, 7 , and 9 in carcinogenesis of pancreatic ductal adenocarcinoma. Dis Markers 2016: 9895721, 2016.

13. Kreijne JE, van der Giessen J, Verhaar AP, Peppelenbosch MP, de Vries AC, van der Woude CJ and Fuhler GM: Fecal matrix metalloproteinase- 9 measurement for optimizing detection of disease activity in inflammatory bowel disease. J Clin Gastroenterol 53: 395-397, 2019.

14. Godefroy E, Gallois A, Idoyaga J, Merad M, Tung N, Monu N, Saenger Y, Fu Y, Ravindran R, Pulendran B, et al: Activation of toll-like receptor-2 by endogenous matrix metalloproteinase-2 modulates dendritic-cell-mediated inflammatory responses. Cell Rep 9: 1856-1870, 2014.

15. Wang J, Li Y and Qi Y: Effect of glutamine-enriched nutritional support on intestinal mucosal barrier function, MMP-2, MMP-9 and immune function in patients with advanced gastric cancer during perioperative chemotherapy. Oncol Lett 14:3606-3610, 2017.

16. Langers AM, Verspaget HW, Hawinkels LJ, Kubben FJ, van Duijn W, van der Reijden JJ, Hardwick JC, Hommes DW and Sier CF: MMP-2 and MMP-9 in normal mucosa are independently associated with outcome of colorectal cancer patients. Br J Cancer 106: 1495-1498, 2012.

17. Park MS, Martini WZ, Dubick MA, Salinas J, Butenas S, Kheirabadi BS, Pusateri AE, Vos JA, Guymon CH, Wolf SE, et al: Thromboelastography as a better indicator of hypercoagulable state after injury than prothrombin time or activated partial thromboplastin time. J Trauma 67: 266-276, 2009. 
18. Nguyen NH, Fumery M, Dulai PS, Prokop LJ, Sandborn WJ, Murad MH and Singh S: Comparative efficacy and tolerability of pharmacological agents for management of mild to moderate ulcerative colitis: A systematic review and network meta-analyses. Lancet Gastroenterol Hepatol 3: 742-753, 2018.

19. Abraham C and Cho JH: Inflammatory bowel disease. N Engl J Med 361: 2066-2078, 2009.

20. Silverberg MS, Satsangi J, Ahmad T, Arnott ID, Bernstein CN, Brant SR, Caprilli R, Colombel JF, Gasche C, Geboes K, et al: Toward an integrated clinical, molecular and serological classification of inflammatory bowel disease: Report of a Working Party of the 2005 Montreal World Congress of Gastroenterology. Can J Gastroenterol 19 (Suppl A): A5-A36, 2005.

21. 17. Soop M, Nygren J, Thorell A and Ljungqvist O: Stress-induced insulin resistance: Recent developments. Curr Opin Clin Nutr Metab Care 10: 181-186, 2007.

22. Altshuler AE, Penn AH, Yang JA, Kim GR and SchmidSchönbein GW: Protease activity increases in plasma, peritoneal fluid, and vital organs after hemorrhagic shock in rats. PLoS One 7: e32672, 2012.
23. Brohi K, Singh J, Heron M and Coats T: Acute traumatic coagulopathy. J Trauma 54: 1127-1130, 2003.

24. Lu H, Liu H, Wang J, Shen J, Weng S, Han L, Sun T, Qian L, Wu M, Zhu S, et al: The chemokine CXCL9 exacerbates chemotherapy-induced acute intestinal damage through inhibition of mucosal restitution. J Cancer Res Clin Oncol 141: 983-992, 2015.

25. Gao Q, Meijer MJ, Schlüter UG, van Hogezand RA, van der Zon JM, van den Berg M, van Duijn W, Lamers CB and Verspaget HW: Infliximab treatment influences the serological expression of matrix metalloproteinase (MMP)-2 and -9 in Crohn's disease. Inflamm Bowel Dis 13: 693-702, 2007.

26. Li WL, Wu CH, Yang J, Tang M, Chen LJ and Zhao SL: Local inflammation alters MMP-2 and MMP-9 gelatinase expression associated with the severity of nifedipine-induced gingival overgrowth: A rat model study. Inflammation 38: 1517-1528, 2015.

(c) (i) () This work is licensed under a Creative Commons

GY No No Attribution-NonCommercial-NoDerivatives 4.0 International (CC BY-NC-ND 4.0) License. 